\title{
Key stages of material expansion in dielectrics upon femtosecond laser ablation revealed by double- color illumination time-resolved microscopy
}

\author{
Mario Garcia-Lechuga ${ }^{1,2}$, Javier Solis ${ }^{1}$, and Jan Siegel ${ }^{1}$ \\ ${ }^{1}$ Laser Processing Group, Instituto de Optica, Consejo Superior de Investigaciones Científica (IO, \\ CSIC), Serrano 121, 28006 Madrid, Spain \\ ${ }^{2}$ Current affiliation: Centro de Láseres Pulsados, CLPU, Parque Científico, 37185 Villamayor, \\ Salamanca, Spain \\ Emails: garcialechuga.mario@gmail.com, j.siegel@io.cfmac.csic.es
}

\begin{abstract}
The physical origin of material removal in dielectrics upon femtosecond laser pulse irradiation (800 nm, $120 \mathrm{fs}$ pulse duration) has been investigated at fluences slightly above ablation threshold. Making use of a versatile pump-probe microscopy set-up, the dynamics and different key stages of the ablation process in lithium niobate have been monitored. The use of two different illumination wavelengths, $400 \mathrm{~nm}$ and $800 \mathrm{~nm}$, and a rigorous image analysis combined with theoretical modelling, enables drawing a clear picture of the material excitation and expansion stages. Immediately after excitation, a dense electron plasma is generated. Few picoseconds later, direct evidence of a rarefaction wave propagating into the bulk is obtained, with an estimated speed of $3650 \mathrm{~m} / \mathrm{s}$. This process marks the onset of material expansion, which is confirmed by the appearance of transient Newton rings, which dynamically change during the expansion up to approximately $1 \mathrm{~ns}$. Exploring delays up to 15 $\mathrm{ns}$, a second dynamic Newton ring pattern is observed, consistent with the formation of a second ablation front propagating five times slower than the first one.
\end{abstract}




\section{Introduction}

Material removal, also termed ablation, induced upon irradiation with femtosecond laser pulses is a topic of increasing interest in the industrial domain [1]. Its main advantage compared to more conventional material removal techniques or to irradiation with nanosecond laser pulses, is the excellent control of the excited region, as well as the high quality of the modifications achieved, caused by a considerable reduction of thermal load effects. From a fundamental point of view, fs-laser ablation has evolved into a very active and broad field of research over the last decades, since it connects to different fields such as nonlinear optics, plasma physics, solid-state physics or non-equilibrium thermodynamics through processes that are present during laser-matter interaction [2].

Among many pioneering experimental studies on this topic, those of $\mathrm{D}$. von der Linde, $\mathrm{K}$. Sokolowski-Tinten and coworkers $[3,4]$ provided the key for understanding the ablation process in metals and semiconductors at moderate fluences. By exploiting the full potential of a pump-probe imaging technique, firstly proposed by Downer et al [5], they observed the formation and dynamic evolution of a transient Newton ring pattern in the nanosecond and sub-nanosecond time range after excitation. This pattern, formed by interference of the probe beam reflected at the front and back interfaces of the semi-transparent ablating layer, allowed a characterization of the propagation speed and its fluence dependence, reaching values up to $1 \mathrm{~km} / \mathrm{s}$. Supported by theoretical modelling [6,7], the thermodynamic scenario is the following [8]: Immediately after excitation, a thin layer is isochorically heated up to temperatures and pressures well above the critical point. Then, adiabatic expansion of this layer occurs in the form of a propagating rarefaction wave travelling from the surface into the bulk, which is reflected upon reaching the cold substrate (rigid wall) causing an inversion of the propagation direction. During the expansion of the layer, the system enters the liquid-gas coexistence region, which causes the speed of the rarefaction wave to be abruptly reduced. As a consequence, the expanding material adopts a shell-like configuration, with a dense shell at liquid-density and an inner volume formed by a liquid-gas mixture.

While the validity of this scenario had been demonstrated only for metals and semiconductors, recently our group could extend it to several dielectrics [9], also employing pump-probe microscopy. The high reflectivity contrast of the Newton rings observed, with values well above the initial reflectivity, allowed to experimentally demonstrate and temporally characterize the transition from a single homogeneous layer into a shell structure. This discovery illustrates the high potential of time-resolved imaging techniques to capture a variety of transient physical processes taking place in laser-excited transparent materials. This fact is demonstrated by the recent characterization of exciting phenomena, such as the formation of a heat-affected-layer [10] or light amplification in dielectrics upon excitation [11], and accompanied by the development of new imaging strategies [12-14].

In the present work, the aim is to provide a comprehensive characterization of the excitation and ablation processes occurring in dielectrics, by employing two different probe wavelengths to monitor the material evolution. The dielectric material chosen for this study is lithium niobate, which has been shown to be especially suitable for the study of the ablation dynamics [15] in dielectrics, given that its high refractive index ensures a high contrast in the observation of different transformation stages.

\section{Experimental configuration}

The femtosecond time-resolved imaging technique used in this work combines an optical pump -probe technique together with a microscopy system. A laser system (Spitfire Pro, 
Spectra Physics) produces linearly polarized pulses of 120 fs FWHM (Full Width at Half Maximum) at $800 \mathrm{~nm}$ and $100 \mathrm{~Hz}$ repetition rate, which are used as pump and probe pulses.

In Figure 1, a sketch of the experimental set-up is shown. A mechanical shutter, synchronized with the laser system, enables to select single pulses. By means of a beam splitter, $70 \%$ of the total pulse energy is selected for the pump pulse (irradiation) and $30 \%$ for the probe pulse (illumination). The pump pulse energy is controlled with a rotating half-wave plate and a polarizing cube, with only vertically polarized light passing ( $\mathrm{Y}$-axis direction, see Figure 1 ). The incident pulse energy is monitored by means of a fast photodiode, which is calibrated with respect to a power meter, placed at the sample position. The pump pulse passes through a motorized optical delay line, enabling the control of the temporal delay between pump and probe pulses, with a resolution of a few tens of fs. Finally, the pump pulse is focused with a lens $\left(\mathrm{f}=150 \mathrm{~mm}\right.$ ) onto the sample surface at an angle of incidence of $52^{\circ}$, leading to an elliptical excitation spot with diameters of $2 \omega_{x}=98.4 \mu \mathrm{m}$ and $2 \omega_{y}=59.0 \mu \mathrm{m}$ (characterized with the methodology introduced by Liu [16]).

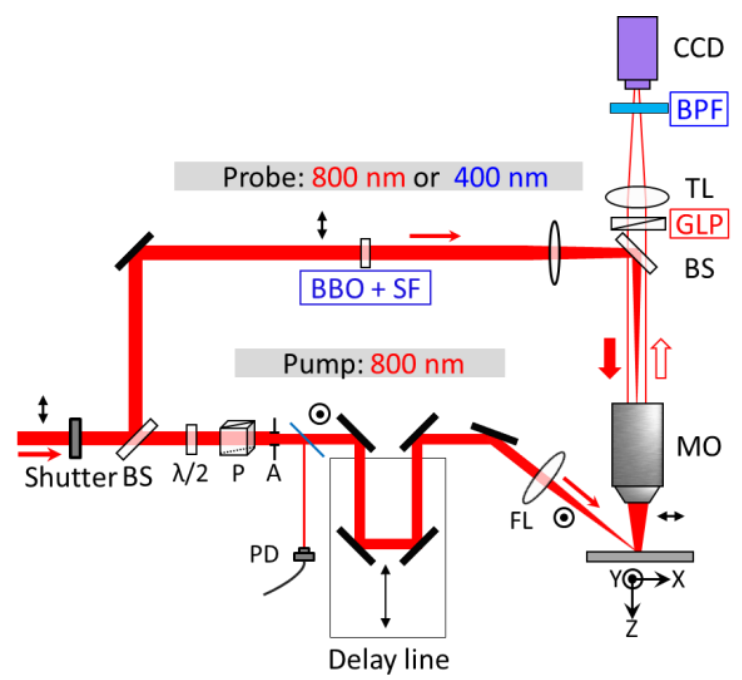

Figure 1 Sketch of the irradiation and time-resolved microcopy setup. Black arrows and circles with a dot indicate horizontal ( $\mathrm{X}-\mathrm{Z}$ plane) and vertical ( $\mathrm{Y}$-axis) polarization respectively. Blue-marked and red-marked elements are respectively used for $400 \mathrm{~nm}$ or $800 \mathrm{~nm}$ illumination wavelength. BS: Beam Splitter; $\lambda / 2$ : HalfWave Plate; P: Polarizing Beam Splitter; A: Aperture; PD: Photo Diode; FL: Focusing Lens; BBO: $\beta$-Barium Borate crystal; SF: Shortpass Filter MO: Microscope Objective; GLP: Glan-Laser Polarizer; TL: Tube Lens; BPF: Band-Pass Filter; CCD: Charge-Coupled Device (Camera).

The probe pulse wavelength used for illumination is either $800 \mathrm{~nm}$ or $400 \mathrm{~nm}$, and is directed onto the imaging axis by means of a $50-50$ beam splitter with a broad spectral working range (350-1100 nm). In order to generate a large illuminated field at the sample surface, the beam is focused before the entrance of the microscope objective $(80 x, N A=0.5)$. The light reflected at the sample surface is collected by the objective and imaged onto a charge-coupled device (CCD) camera employing a tube lens.

Illumination at $400 \mathrm{~nm}$ is produced by frequency doubling the fundamental laser wavelength using a beta barium borate crystal (BBO) and a short pass filter. Additionally, a band-pass filter is placed in front of the CCD to block scatter from the pump pulse $(800 \mathrm{~nm})$. Illuminating at 800 $\mathrm{nm}$ is challenging, since discrimination between pump and probe light cannot be done spectrally. In this case, blocking of scattered pump light is performed by polarization discrimination, since the pump and probe pulses are orthogonally polarized. To this end, a high-extinction-ratio Glan-Laser polarizer is placed just before the tube lens. However, scattered pump light is partially depolarized [17], which reduces the effective extinction ratio of the polarizer. For that reason, the illumination intensity was increased to enhance the 
signal-to-noise ratio of the probe light. It was tested that this intensity increase of the illumination beam does not affect the final modification produced in the material, neither on its own nor in combination with the pump pulse.

The temporal resolution of the two color experiments has been determined by measuring the temporal cross-correlation signal between the pump and probe pulses. In the case of $400 \mathrm{~nm}$ illumination, a UV-photodiode (SIC01M, Roithner) is placed at the sample position, yielding a cross-correlation curve with a FWHM of $310 \mathrm{fs}$. This temporal resolution is slightly less than in our previous works where the experimental configuration included a prism compressor for dispersion compensation (cross-correlation curve of $200 \mathrm{fs}$ ) $[15,18]$. In the case of $800 \mathrm{~nm}$ illumination, we used the experimentally measured FWHM of the pump pulse (120 fs), as measured by FROG to estimate a cross-correlation value of $170 \mathrm{fs}$, assuming negligible dispersion of the optical elements at this wavelength. With regards the temporal resolution, it must be noticed that although the crossed beam geometry of the experiment introduces some temporal smearing due to the landing time of the pump pulse, for a given a spatial location on the irradiated spot, this effect is much smaller than dispersion and cross correlation effects over the experiment resolution.

It should be noted that each time-resolved image was recorded upon irradiation with a single laser pulse, at a fixed time delay between pump and probe pulse and irradiating an unexposed surface. Before irradiation, an image of the unexposed surface is recorded upon illumination with a probe pulse, which is used to normalize the image recorded upon pump excitation. That way, the normalized images provide information on the transient changes in reflectivity, with the relative reflectivity $\left(R_{r e l}\right)$ of the non-excited surface being equal to 1 .

The spatial dimension of the images together with the Gaussian energy profile of the laser beam, allows accessing the material response under different local fluences after a single pulse irradiation with a peak fluence equal to $F_{0}$. For obtaining the corresponding local fluence at a given distance from the center $(\Delta x)$ the following formula is used: $F(\Delta x)=F_{0} e^{-2 \Delta x^{2} / \omega_{x}^{2}}$.

The sample used in this study is a commercial, $2 \mathrm{~cm} \times 1 \mathrm{~cm} \times 1 \mathrm{~cm}$, single crystal of lithium niobate supplied by VM-TIM Germany, with all surfaces fine-polished. Irradiations were performed at the face perpendicular the c-axis, where the ordinary and extraordinary refractive indices are the same.

\section{Results and discussion}

Figure 2(a) displays selected time-resolved images recorded at different delays after single pulse excitation at a peak fluence of $F_{0}=2.05 \mathrm{~J} / \mathrm{cm}^{2}$. The final modification of the surface topography produced can be seen in Figure 2 (b), showing a maximum crater depth of $100 \mathrm{~nm}$ and a small surface protrusion at the crater edge with a maximum height less than $10 \mathrm{~nm}$. The modification threshold is found to be $F_{t h}=1.55 \mathrm{~J} / \mathrm{cm}^{2}$, by using the Liu method [16]. Images recorded at the same time delays under the two illumination wavelengths are shown, enabling a direct comparison of the different reflectivity behavior. In the following sections, a detailed study of the reflectivity evolution over different temporal windows and the associated physical processes is presented. 

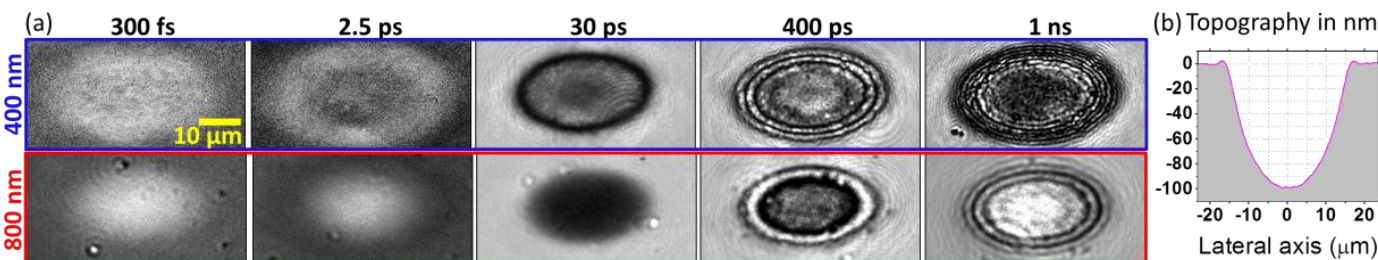

Figure 2 (a) Time-resolved microscopy images recorded at different delays (labels) after irradiation with single $120 \mathrm{fs}, 800 \mathrm{~nm}$ pulses at a peak fluence $F_{0}=2.05 \mathrm{~J} / \mathrm{cm}^{2}$, recorded at $400 \mathrm{~nm}$ (upper row) and $800 \mathrm{~nm}$ (lower row) illumination wavelengths. (b) Vertical profile of the final ablation crater produced, measured with a whitelight-interferometer microscope (model Sensofar Pl $\mu$ 2300, 50x objective lens (N.A. $=0.55$ ), lateral resolution $\sim 600 \mathrm{~nm}$, vertical resolution, $<1 \mathrm{~nm}$.)

3.1 Free electron generation: estimation of the maximum electron density and electronelectron scattering rate.

In Figure $3(\mathrm{a})$, the evolution of the reflectivity in the center of the excited region, where the local fluence corresponds to the peak fluence $\left(F=2.05 \mathrm{~J} / \mathrm{cm}^{2}\right)$, is shown for ultrashort time delays $(<1 \mathrm{ps})$. For this relatively high local fluence, as shown in ref.[15], electron plasma dominate over Kerr response effects in the material response at zero delay. The observed increase in reflectivity for both illumination wavelengths is associated to a transient semimetallic behavior of the material, caused by the generation of a high density of free electrons via multiphoton and impact ionization $[19,20]$.
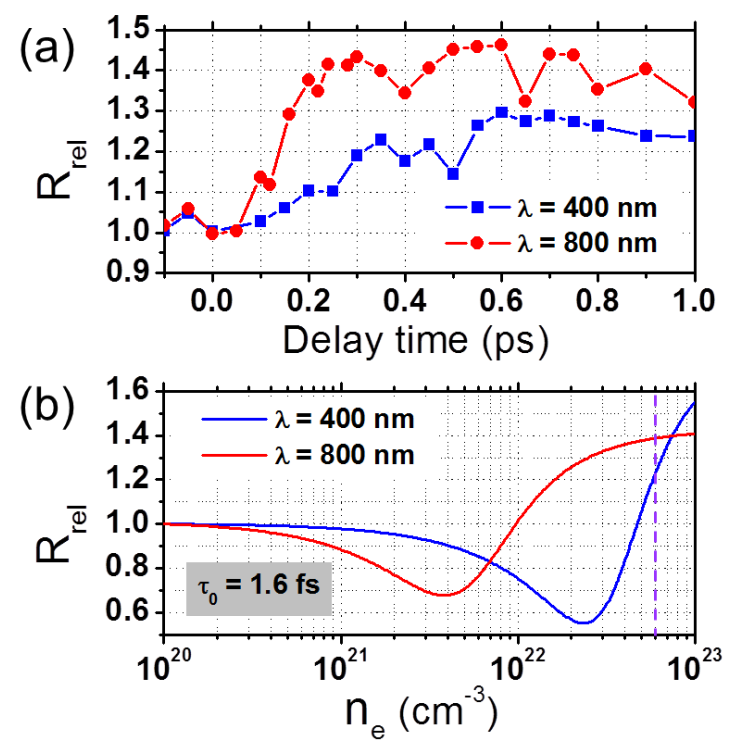

Figure 3 (a) Measured reflectivity evolution in the center of the excited region $\left(F_{0}=2.05 \mathrm{~J} / \mathrm{cm}^{2}\right)$ at two different illumination wavelengths: $400 \mathrm{~nm}$ and $800 \mathrm{~nm}$. (b) Calculated evolution of the reflectivity at two different illumination wavelengths as a function of the free electron density following the Drude model (see text). The dashed vertical line (purple) corresponds to the maximum free electron density that best fits the maximum reflectivity values observed experimentally for both probe wavelengths.

While the excitation conditions are the same for both illumination wavelengths, the maximum reflectivity values are reached at different time delays: after $200 \mathrm{fs}$ for $800 \mathrm{~nm}$ and after $600 \mathrm{fs}$ for $400 \mathrm{~nm}$. Part of this slower rise at $400 \mathrm{~nm}$ could be caused by a slower instrumental response function (see experimental section, cross correlation $=310 \mathrm{fs}$ ). However the faster rise when illuminating at $800 \mathrm{~nm}$ could also be attributed to a higher sensitivity to lower free electron densities, as shown in Figure 3 (b), by performing Drude model calculations.

This model, discussed in further in detail in ref [21], relates the change in the complex refractive index $(\tilde{n})$ of the surface material to the change in free electron density $\left(n_{e}\right)$, 


$$
\tilde{n}^{2}=\tilde{n}_{0}^{2}-\frac{n_{e}}{n_{c}} \cdot \frac{1}{1+i(\omega \tau)^{-1}}
$$

where $\tilde{n}_{0}$ is the complex refractive index of the non-excited material, $\omega$ the illumination light frequency, $n_{c}$ the electron critical density $\left(n_{c}=m^{*} \epsilon_{0} \omega^{2} / e^{2}\right.$, with $e$ electron charge and $m^{*}$ the reduced electron mass), and $\tau$ the electron-electron collision time or damping constant, whose evolution can be expressed in a simplified approach as being inversely proportional to the photo-generated carrier density, $\tau=\tau_{0} n_{c} / n_{e}$, as reported in Refs. [22,23], being $\tau_{0}$ the damping constant without laser excitation. This change in refractive index can be translated into a change in reflectivity by applying Fresnel equations. Since the incident media in our case is air and the illumination incidence is normal to the surface, the formula is simplified to the following expression,

$$
R_{r e l}\left(n_{e}\right)=\frac{R\left(n_{e}\right)}{R_{0}}=\left|\frac{\tilde{n}-1}{\tilde{n}+1}\right|^{2} /\left|\frac{\tilde{n}_{0}-1}{\tilde{n}_{0}+1}\right|^{2}
$$

where $R_{0}$ is the reflectivity of the non-excited surface. In particular in Figure 3 (b) Drude model calculation are shown for both illumination wavelengths, using the optical constants of lithium niobate: $n_{0}(400 \mathrm{~nm})=2.439$ and $n_{0}(800 \mathrm{~nm})=2.255$ [24], and assuming $m^{*}=m_{e}$ (the electron mass), as done in ref. [25] Since the reflectivity for $800 \mathrm{~nm}$ illumination seems to saturate, attaining a lower value $\mathrm{R}_{\text {rel }} \sim 1.4$, a scattering time value equal to $\tau_{0}=1.6 \mathrm{fs}$ was estimated in order to reproduce this behavior.

Regarding the Drude model calculation in Figure 3 (b), it has to be noted that the predicted initial reflectivity decrease is not observed experimentally. We recently reported in a study on fused silica and sapphire [26] the underlying reason for the absence of such pronounced reflectivity dip. In that study, employing a more complex model including multiple-rate equations (MRE), it was shown that a deviation from the simple scaling law of the scattering rate with the electron density during the initial phase of the excitation process leads to a modification of transient reflectivity that departs from the basic Drude model. However, it was also shown in Ref [26] that once the critical density is exceeded, the MRE model and Drude model with the simple $\tau$ scaling show similar behavior. Accordingly, we can estimate that the maximum electron density reached, by associating the reflectivity maxima experimentally observed in Figure $3(a)\left(R_{\text {rel }}=1.25\right.$ and $R_{\text {rel }}=1.4$ for $400 \mathrm{~nm}$ and $800 \mathrm{~nm}$, respectively) with the Drude model calculation, is $6 \cdot 10^{22} \mathrm{~cm}^{-3}$, as marked with a dashed line in Figure 3 (b). We would like to stress that the reflectivity at $400 \mathrm{~nm}$ does not saturate under the present irradiation conditions: a higher maximum reflectivity at $400 \mathrm{~nm}$ is reached by exciting at higher fluences. We have verified this by performing irradiations at $4.6 \mathrm{~J} / \mathrm{cm}^{2}$ reaching reflectivities up to $R_{\text {rel }}=1.6$. Yet, the reflectivity saturates for $800 \mathrm{~nm}$ in agreement with the Drude model estimations above shown.

\subsection{Free electron plasma relaxation and propagation of a rarefaction wave}

As already observed by visual inspection in Figure 2 the reflectivity behavior strongly depends on the illumination wavelength, as well as on the local position of the excited region (directly related to the local fluence [22]). In Figure 4, this dependency is illustrated, showing the evolution of the reflectivity from 0 to 50 ps for three different local fluences, and at both illumination wavelengths.

Overall, the reflectivity decreases strongly, which is related in part to the reduction on the free electron density produced by inelastic scattering with the lattice, among other mechanisms, resulting in the generation of phonons. Since the irradiation conditions are identical, this reduction of the free electron density is the same for both experiments, using different probe wavelengths. However, it can be seen that the reflectivity drops below the initial value $\left(R_{\text {rel }}<1\right)$ at different times; namely after 3 ps for $400 \mathrm{~nm}$ and after 8-10 ps for $800 \mathrm{~nm}$. 
The strong decrease of the normalized reflectivity well below one cannot be explained by taking into account the free electron density decrease alone, but it is a signature of surface ablation. As explained by the hydrodynamic model of material ablation [8], isochoric heating of the thin laser-excited layer is produced by electron-phonon scattering. This layer reaches an extremely hot, high pressure state, and features an optical behavior similar to a black-body within the range $10-30 \mathrm{ps}$, as we discussed in Ref [26], since we also observe a simultaneous decrease of the sample transmittivity (data not shown). Yet, the verification of the existence of a transient black body would require measurements of the transient evolution of the light emission of the surface.

Even if the mechanisms leading to the strong reflectivity decrease are not completely clear, there is a relevant aspect that should be pointed out: the lack of dependency on the local fluence. For both wavelengths, especially for the data recorded at $400 \mathrm{~nm}$ illumination, this effect is evident in the range from 4 ps to $12 \mathrm{ps}$, for local fluences of $2.05,1.88$ and $1.79 \mathrm{~J} / \mathrm{cm}^{2}$.

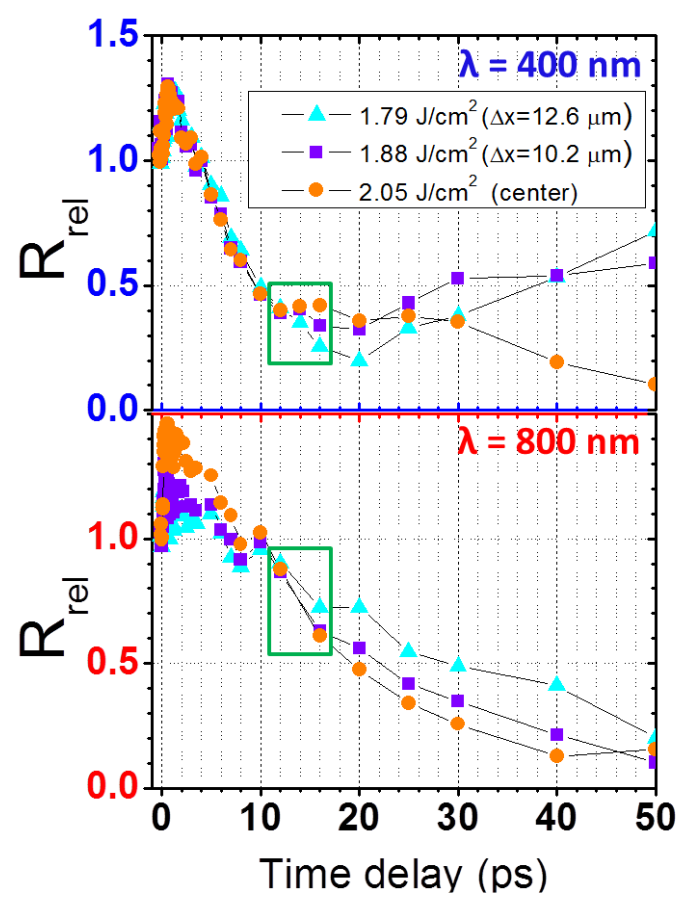

Figure 4 Evolution of local reflectivity extracted from the images at three different positions of the excited region (three different local fluences), being $\Delta x$ the distance from the center of the irradiation. The upper frame corresponds to the analysis of the images recorded at $400 \mathrm{~nm}$ and the lower frame to the ones recorded at 800 $\mathrm{nm}$. The green rectangle marks the time window when a change in the reflectivity tendency for the different local fluences is produced.

The observed monotonous reflectivity decrease, independent of the local fluence, is the first direct experimental evidence of a propagating rarefaction wave produced after isochoric heating, which firstly propagates from the surface into the bulk at the speed of sound of the material $\left(c_{0}\right)$, related to the adiabatic expansion as proposed by the thermodynamic model of material ablation [8]. The propagation speed of the rarefaction wave is independent of the local fluence, having as a consequence that modified layer has the same density profile (gradient) and for instance the same optical properties at different lateral positions of the irradiated region.

However the optical properties at different positions change when the rarefaction wave is reflected at the interface of the heated layer with the substrate, which depends on the thickness of heated material that is indeed a fluence-dependent factor. It is therefore possible to associate the change of slope in the reflectivity decay for the different fluences, marked with green rectangles in Figure 4, with the reflection of the rarefaction wave at different 
depths for different lateral positions. This statement is based on the observation that the first trend change occurs for the lowest fluence studied, $1.79 \mathrm{~J} / \mathrm{cm}^{2}$, where the heated layer $(d)$ is thinner compared to higher fluences and as a consequence the rarefaction wave arrives earlier at the interface with the substrate $\left(t=d / c_{0}\right)$. It is also worth noting that the relative reflectivity values for different fluences are inverted between 15 and 25 ps for the two wavelengths, i.e. the reflectivity increases with fluence for $400 \mathrm{~nm}$ but decreases for $800 \mathrm{~nm}$, which further underlines the interference nature of these reflectivity changes, leading to either a reflectivity maximum or minimum depending on the observation wavelength. By correlating the shape and thickness of the heated layer in a first approximation with the one of the final crater (shown in Figure 2 (b)), an estimation of the propagation speed of the rarefaction wave can be obtained. The first slope change occurs at $14 \mathrm{ps}$ for $1.79 \mathrm{~J} / \mathrm{cm}^{2}(\Delta \mathrm{x}=12.6 \mu \mathrm{m})$, where the crater has a depth of $45 \mathrm{~nm}$, obtaining: $c_{0}=45 \mathrm{~nm} / 14 \mathrm{ps}=3200 \mathrm{~m} / \mathrm{s}$. For $1.88 \mathrm{~J} / \mathrm{cm}^{2}(\Delta \mathrm{x}=10.2 \mu \mathrm{m})$, the trend change occurs at $16 \mathrm{ps}$, yielding $c_{0}=65 \mathrm{~nm} / 16 \mathrm{ps}=4100 \mathrm{~m} / \mathrm{s}$. Taking into account the relatively large error of this measurement, the values are consistent with a constant sound velocity of $c_{0} \approx 3650 \mathrm{~m} / \mathrm{s}$. This value is in good agreement with values reported in literature for the velocity of the acoustic waves in non-excited $\mathrm{LiNbO}_{3}$, with values in the $3500-4000 \mathrm{~m} / \mathrm{s}$ $[27,28]$ range for the crystallography direction of our study (c-cut).

Following this value, the reflection of the rarefaction at the center of the irradiated spot $(\Delta x=0$ $\mu \mathrm{m}, 2.05 \mathrm{~J} / \mathrm{cm}^{2}, \mathrm{~d}=100 \mathrm{~nm}$ ) should occur for a time delay of $25-28 \mathrm{ps}$. At this time delay two sharp interfaces are formed $[6,8]$, which leads to the formation of high contrast constructive and destructive interferences, as observed with the pronounced decreased of the reflectivity curve in Fig. 4 , corresponding to a destructive interference.

\subsection{Material expansion: observation of two consecutive Newton rings patterns}

In Figure 2, a ring structure was observed from 30 ps onwards, with the number of rings increasing with time. These so-called transient Newton rings are formed by optical interference of the illumination beam reflected at the front and back surfaces of the expanding layer [3]. Bright regions correspond to constructive interference, which are produced when the optical path difference between the two surfaces is equal to $\lambda$ or a multiple of it $(\# \cdot \lambda)$. This means that a bright central disk of the excited region corresponds to an optical layer thickness $\mathrm{D}=\# \cdot \lambda / 2$, with $\mathrm{D}=d_{\text {layer }} \cdot n$. As an example, for the image recorded at $400 \mathrm{~nm}$ illumination at a delay of 400 ps shown in Figure 2, one can appreciate 4 constructive interference events (CIE) in form of 3 rings and 1 disk, compared to $2 \mathrm{CIE}$ at $800 \mathrm{~nm}$. According to the relation described above, this consistently yields for both wavelengths a maximum optical layer thickness of $D=$ $800 \mathrm{~nm}$.

Performing this analysis for a series of images recorded over a wide delay time interval, the temporal evolution of maximum optical layer thickness can be extracted, as shown in Figure 5. The data obtained for both illumination wavelengths follows a clear linear behavior for time delays $>200$ ps. This minimum delay effect can be attributed to the time it takes to develop the final structure of the expanding layer: a thin shell (liquid density) enclosing a low-density region (liquid-vapor coexistence), as theoretically proposed by Anisimov and coworkers [6] and experimentally demonstrated for dielectrics in our recent work [9]. A linear fit to the data for delays above $200 \mathrm{ps}$ yields a slope of $1400 \mathrm{~m} / \mathrm{s}$. This value corresponds to the product of the expansion velocity $(v)$ times the effective refractive index of the bubble-like structure $\left(n_{\text {eff }}\right)$. The obtained value is higher than the one we reported in [9] $(1140 \mathrm{~m} / \mathrm{s})$, which can be explained by the higher peak fluence used here. 


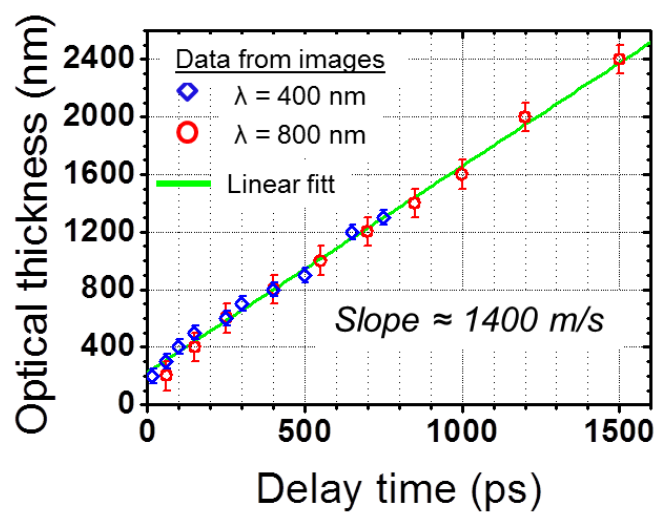

Figure 5 Temporal evolution of the optical thickness (D) of the expanding layer in its center. The data has been obtained from the number of constructive and destructive interference events in the Newton ring patterns. A linear fit has been performed for delays above 200 ps.

Given that the numbers of rings for $800 \mathrm{~nm}$ illumination is inherently lower, a characterization of the layer expansion is possible up to relatively long delays, before a too high ring density prevents a reliable image analysis, as shown in Figure 5. During this exploration of long delays, a surprising phenomenon was observed, in form of a pulsating reflectivity modulation on a longer time scale, superimposed onto the ring pattern. As illustrated in Figure 6 (a), at 1 ns there are $4 \mathrm{CIE}$, accompanied by a high reflectivity in the center $\left(R_{\text {rel }} \sim 1.5\right)$, while at 1.5 ns there are $6 \mathrm{CIE}$, accompanied by a low reflectivity in the center $\left(R_{\text {rel }} \sim 0.5\right)$. Again, at $2.5 \mathrm{~ns}$ a pronounced increase in reflectivity in the spot center is observed.

The complete set of images shown in Figure 6 (a) demonstrates that this second modulation corresponds to a second Newton ring pattern, not previously reported. By analyzing the reflectivity in the center of a large series of such images, the reflectivity evolution up to 9 ns has been extracted, as shown in Figure 6 (b). Even though the data suffers from noise, a clear periodic oscillation can be observed for $800 \mathrm{~nm}$ wavelength, with a period of approximately $1.5 \mathrm{~ns}$. According to the relation above described, this corresponds to an expansion velocity for this secondary front of $\approx(270 \mathrm{~m} / \mathrm{s}) / n_{2, \text { eff }}$, with $n_{2, \text { eff }}$ being the (unknown) effective refractive index of this secondary expanding layer. The reflectivity contrast in Figure 6 (b) is determined to lie between $R_{\text {rel }} \sim 1.5$ and 0.4 . Such high contrast, with values well above the initial reflectivity, cannot be simulated assuming a homogeneous refractive index of the layer. Similarly to the first Newton ring pattern, characterized in detail in Ref. [9], also this second and slower expanding layer is therefore expected to have a shell-like structure.

Combing the information extracted from the observation and characterization of the two Newton ring patterns, we propose an ablation scenario as shown in the sketch of Figure 6 (c), relating the second pattern to the presence a secondary delayed ablation front. We attribute the absence of this slow reflectivity modulation for $400 \mathrm{~nm}$ illumination (c.f. Figure 6 (a)) to a stronger Rayleigh scattering at short wavelengths. Rayleigh scattering is expected to occur inside the shell of the secondary expanding layer due to a presumably higher content of larger size liquid droplets (or vapor bubbles) during the secondary expansion.

Even though a second transient Newton ring pattern has not been observed before in any material, evidence of the existence of secondary ablation processes was previously reported. Zhang and coworkers [29] employed time-resolved shadowgraphy to monitor material ejection in an intermittent process, when irradiating metals and semiconductors surfaces at very high fluences $\left(40 \mathrm{~J} / \mathrm{cm}^{2}\right)$. The authors attributed this phenomenon to the compression of the material by stress waves formed after expansion of the first front, leading to secondary ablation, once the repelling force surpasses the compressed stress. Hu and coworkers [30], employing a similar experimental configuration and the same excitation fluence, observed in 

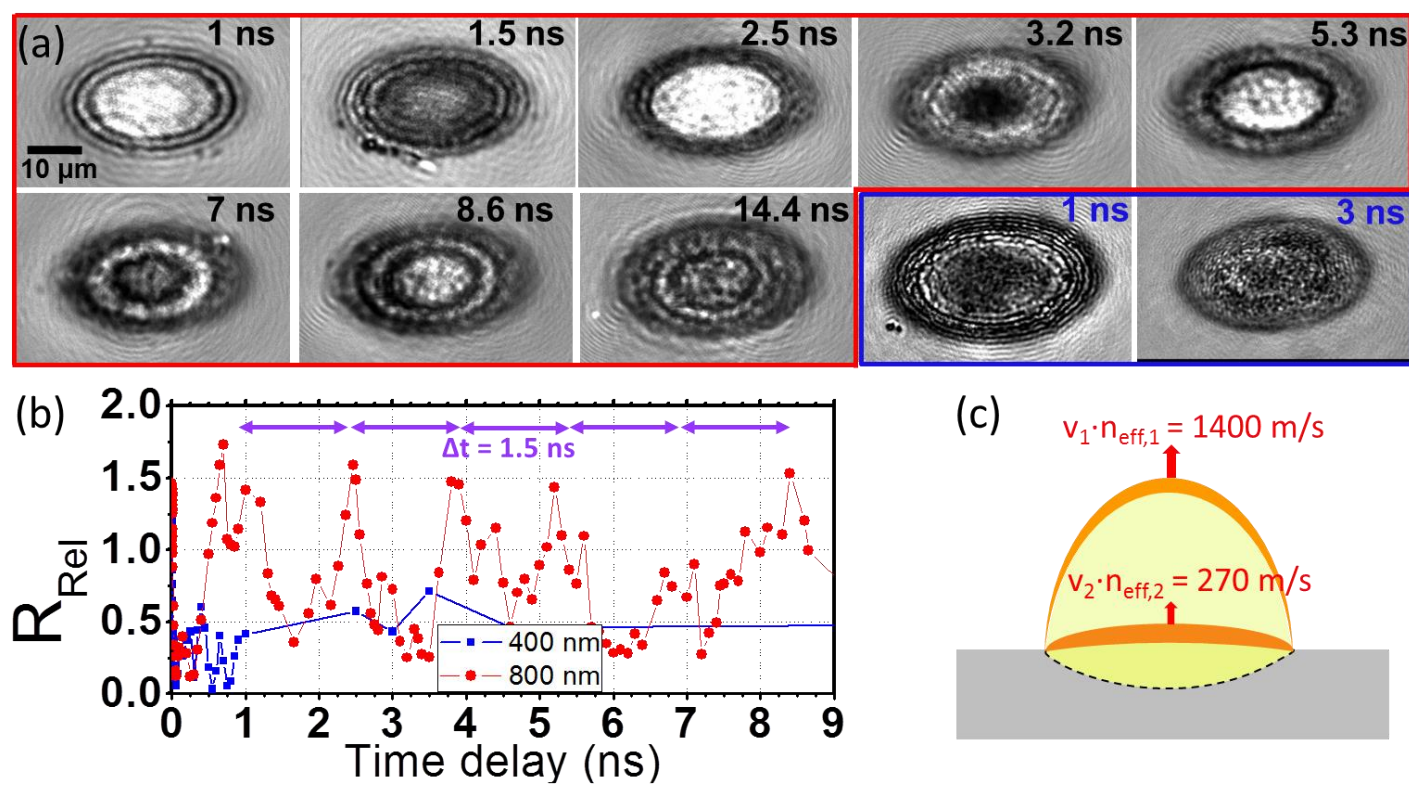

Figure 6 (a) Time-resolved microscopy images recorded under the same irradiation conditions as in Figure 2. The red-framed images were recorded under $800 \mathrm{~nm}$ illumination wavelength, the blue-framed images at 400 $\mathrm{nm}$. (b) Evolution of the reflectivity at the spot center as a function of time. Violet arrows indicate the time separation between two consecutive local maxima. (c) Sketch of the proposed scenario of material expansion represented in a cross-section view.

silica glass two different material ejection processes in the nanosecond time scale, accompanied by the propagation of stress waves inside the bulk. While at first glance these works might suggest similar processes being responsible of the formation of the second Newton ring pattern in our case, the fact that our irradiation fluences are comparatively low and close to the ablation threshold, necessarily implies important differences.

A different plausible explanation for this behavior could be related to the possible propagation of a melt front into the material, forming a layer of transiently molten material, whose thickness increases with time. Even though propagation speed values close to $350 \mathrm{~m} / \mathrm{s}$ for such a melt-front were reported in silver [31], those experiments cannot be directly compared with the present data, because of the different material type (metal vs dielectric) and the configuration in form of a thin film. In a different dielectric, such a phosphate glass, our group has recently reported the observation of a Newton ring pattern related to propagation of a melt-in front [10]. The results allowed to stablish the formation of a melt-in front with a gradually slowing down propagation speed $(1 \mathrm{~m} / \mathrm{s}$ at $5 \mathrm{~ns}$ after excitation), leaving behind a layer with homogenous refractive index. Both findings strongly differ from the those reported here (constant high speed $(270 \mathrm{~m} / \mathrm{s}) / n_{2, \text { eff }}$ and shell-like structure), which suggest that the observed second Newton ring pattern does not correspond to a melt front.

Finally, it might be possible that fragmentation of the main ablation layer into two sub-layers propagating collinearly at different velocities could cause an oscillatory behavior of the reflectivity. Even if this alternative cannot be a priori excluded, a neat reason for such fragmentation of the ablating layer to occur would be required to render this alternative plausible. Further research, both experimental (e.g. in other dielectrics) and theoretical modeling, is needed to ultimately clarify the origin of this second Newton ring pattern.

\section{Conclusions}

As a general conclusion, it is clear that the use of different illumination wavelengths and the exploration of a large temporal window are highly beneficial in time-resolved microscopy experiments, enabling a much more comprehensive characterization of material excitation and 
ablation dynamics. In particular, we have performed a complete study of the transformation dynamics of lithium niobate for fluences slightly above the ablation threshold. At the subpicosecond time scale, the characterization of the reflectivity response combined with calculations using a Drude model allowed us to estimate the maximum free electron density generated and the electron-electron scattering time. Moreover, we provide direct experimental evidence of the propagation of a rarefaction wave into the bulk, with a fluenceindependent propagation speed of $v \approx 3650 \mathrm{~m} / \mathrm{s}$. This experimental observation corroborates the existence of adiabatic expansion, as proposed by the hydrodynamic model of material ablation. Rarefaction wave propagation marks the onset of material expansion and ablation, which has been characterized in detail by the analysis of Newton ring patterns up to delays of a few nanoseconds. Finally, the formation of a second ring pattern, only visible upon illumination at $800 \mathrm{~nm}$, can be attributed to the propagation of a secondary front of ablating material. This secondary front propagates at a speed five times slower than the primary front.

\section{Acknowledgements}

This work has been partly funded by the Spanish Ministry of Economy and Competiveness (Project No. TEC2014-52642-C2-1-R). M.G.-L. acknowledges the FPU (Formación de Profesorado Universitario) Grant No.AP2012-0217 awarded by the Spanish Ministry of Education. We are grateful to Dr. K. Sokolowski-Tinten for the advices on the development of time-resolved microscopy using the same wavelengths for pump and probe pulses.

\section{Bibliography}

1. K. Sugioka and Y. Cheng, Light Sci. Appl. 3, e149 (2014).

2. E. Gamaly, Femtosecond Laser-Matter Interactions Theory, Experiments and Applications (CRC Press, 2011).

3. K. Sokolowski-Tinten, J. Bialkowski, A. Cavalleri, D. von der Linde, A. Oparin, J. Meyer-terVehn, and S. I. Anisimov, Phys. Rev. Lett. 81, 224 (1998).

4. K. Sokolowski-Tinten, J. Bialkowski, A. Cavalleri, M. Boing, H. Schueler, and D. von der Linde, in Proc. SPIE, edited by C. R. Phipps (1998), pp. 46-57.

5. M. C. Downer, R. L. Fork, and C. V. Shank, J. Opt. Soc. Am. B 2, 595 (1985).

6. S. I. Anisimov, N. A. Inogamov, A. M. Oparin, B. Rethfeld, T. Yabe, M. Ogawa, and V. E. Fortov, Appl. Phys. A Mater. Sci. Process. Process. 69, 617 (1999).

7. N. A. Inogamov, Y. V. Petrov, S. I. Anisimov, a. M. Oparin, N. V. Shaposhnikov, D. von der Linde, and J. Meyer-ter-Vehn, J. Exp. Theor. Phys. Lett. 69, 310 (1999).

8. D. von der Linde and K. Sokolowski-Tinten, Appl. Surf. Sci. 154-155, 1 (2000).

9. M. Garcia-Lechuga, J. Siegel, J. Hernandez-Rueda, and J. Solis, Appl. Phys. Lett. 105, 112902 (2014).

10. M. Garcia-Lechuga, J. Solis, and J. Siegel, Appl. Phys. Lett. 108, 171901 (2016).

11. T. Winkler, L. Haahr-Lillevang, C. Sarpe, B. Zielinski, N. Götte, A. Senftleben, P. Balling, and

T. Baumert, Nat. Phys. 1 (2017). 
12. S. Rapp, M. Kaiser, M. Schmidt, and H. P. Huber, Opt. Express 24, 17572 (2016).

13. L. Gallais and S. Monneret, Opt. Lett. 41, 3245 (2016).

14. M. Chanal, V. Y. Fedorov, M. Chambonneau, R. Clady, S. Tzortzakis, and D. Grojo, Nat. Commun. 8, 773 (2017).

15. M. Garcia-Lechuga, J. Siegel, J. Hernandez-Rueda, and J. Solis, J. Appl. Phys. 116, 113502 (2014).

16. J. M. Liu, Opt. Lett. 7, 196 (1982).

17. P. Beckmann and A. Spizzichino, The Scattering of Electromagnetic Waves from Rough Surfaces (Artech House, 1987).

18. J. Hernandez-Rueda, D. Puerto, J. Siegel, M. Galvan-Sosa, and J. Solis, Appl. Surf. Sci. 258, 9389 (2012).

19. B. Rethfeld, Phys. Rev. B 73, 035101 (2006).

20. M. Lebugle, N. Sanner, N. Varkentina, M. Sentis, and O. Utéza, J. Appl. Phys. 116, 063105 (2014).

21. J. Siegel and J. Solis, in Femtosecond Laser Micromach. Photonic Microfluid. Devices Transparent Mater., edited by R. Osellame, G. Cerullo, and R. Ramponi (Springer Berlin Heidelberg, Berlin, Heidelberg, 2012), pp. 19-41.

22. D. Puerto, J. Siegel, W. Gawelda, M. Galvan-Sosa, L. Ehrentraut, J. Bonse, and J. Solis, J. Opt. Soc. Am. B 27, 1065 (2010).

23. C. Quoix, G. Hamoniaux, A. Antonetti, J.-C. Gauthier, J.-P. Geindre, and P. Audebert, J. Quant. Spectrosc. Radiat. Transf. 65, 455 (2000).

24. D. E. Zelmon, D. L. Small, and D. Jundt, J. Opt. Soc. Am. B 14, 3319 (1997).

25. E. G. Gamaly, S. Juodkazis, V. Mizeikis, H. Misawa, A. V. Rode, and W. Krolikowski, Phys. Rev. B 81, 054113 (2010).

26. M. Garcia-Lechuga, L. Haahr-Lillevang, J. Siegel, P. Balling, S. Guizard, and J. Solis, Phys. Rev. B 95, 214114 (2017)

27. D. Ciplys and R. Rimeika, Ultragarsas 3, 14 (1999).

28. Y. Shibata, K. Kaya, K. Akashi, M. Kanai, T. Kawai, and S. Kawai, J. Appl. Phys. 77, 1498 (1995).

29. N. Zhang, X. Zhu, J. Yang, X. Wang, and M. Wang, Phys. Rev. Lett. 99, 2 (2007).

30. H. Hu, X. Wang, H. Zhai, N. Zhang, and P. Wang, Appl. Phys. Lett. 97, 061117 (2010).

31. W. L. Chan, R. S. Averback, D. G. Cahill, and A. Lagoutchev, Phys. Rev. B - Condens. Matter Mater. Phys. 78, 1 (2008). 